\title{
Caracterización de las propiedades en caliente de aceros para la fabricación de tubos
}

\author{
J. Tormo*, A. Hey* y E. Brandaleze*
}

\begin{abstract}
Resumen Resulta relevante comprender el comportamiento mecánico en caliente de los aceros utilizados en la fabricación de tubos sin costura mediante laminadores perforadores. Dicho proceso contempla la perforación de tochos cilíndricos, a través de una reducción diametral crítica, que genera una fractura en el núcleo y facilita la perforación por efecto Mannesmann junto a la laminación transversal. Se presentan resultados de ductilidad en caliente obtenidos mediante ensayos de torsión en el rango de temperatura de interés industrial y se relacionan con la susceptibilidad a la rotura axial y el análisis de la fractura asociada. Las curvas de ductilidad permiten determinar la variación de ductilidad desde la periferia hacia el centro de la barra y el rango de temperatura a la cual disminuye la ductilidad del material.
\end{abstract}

Palabras clave Tubos. Efecto Mannesmann. Ductilidad. Torsión en caliente.

\section{Hot mechanical behaviour of steels for seamless pipes}

\begin{abstract}
It is necessary to understand the mechanical behaviour of the steels used in the rotary piercing mill process, for the manufacture of seamless pipes. This process involves a critic reduction in diameter of the cylindrical solid billet through a crack caused in the centre by Mannesmann and cross roll mill effects. Hot ductility results obtained by torsion tests at industrial interest temperature range are presented in relation with the axial break susceptibility and the associated fracture. The ductility curves let us determine the ductility variation from the periphery toward the centre of the billet and also the range of temperature at which the ductility of the material diminishes.
\end{abstract}

Keywords Tubes. Mannesmann effect. Ductility. Hot torsion.

\section{INTRODUCCIÓN}

Los procesos más modernos de la fabricación de tubos sin costura incluyen, en las primeras etapas de la deformación, la perforación de tochos cilíndricos provenientes de la colada continua. Este proceso se lleva a cabo mediante laminadores perforadores que trabajan sobre la base del principio de la laminación transversal.

Al producirse durante el proceso, la reducción diametral crítica del tocho, se inicia una fractura en el núcleo que permite el proceso de perforación. Esto ocurre por el conocido efecto Mannesmann, que es independiente de la presencia de la punta. En las figuras 1 y 2, se detallan los efectos de la operación de perforado y el estado de tensiones en el centro del tocho.
Resulta conveniente que la cavidad no se forme delante del mandril, ya que la superficie rugosa propia de la fractura al ser laminada puede dar lugar a la formación de defectos. Por esta razón, el mandril se ubica con cierto avance en relación a la garganta que forman los cilindros, de modo que ésta genere la perforación del tocho mientras es
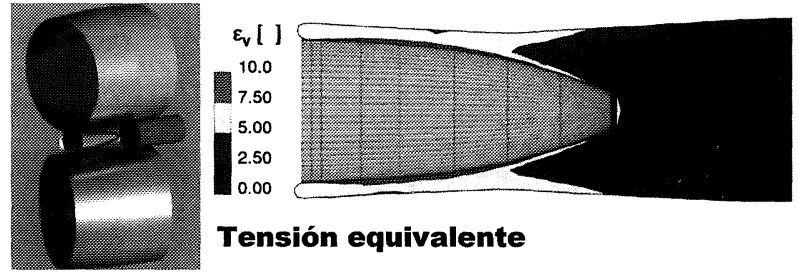

Tensión equivalente

Figura 1. Efectos de la operación de perforado.

Figure 1. Drilling operation effects.

(*) Instituto Argentino de Siderurgia, Avda. Central y 19 Oeste, Barrio Somisa, San Nicolás, Prov. de Buenos Aires, Argentina. Gerencia de Desarrollo - CNEA - Argentina. 


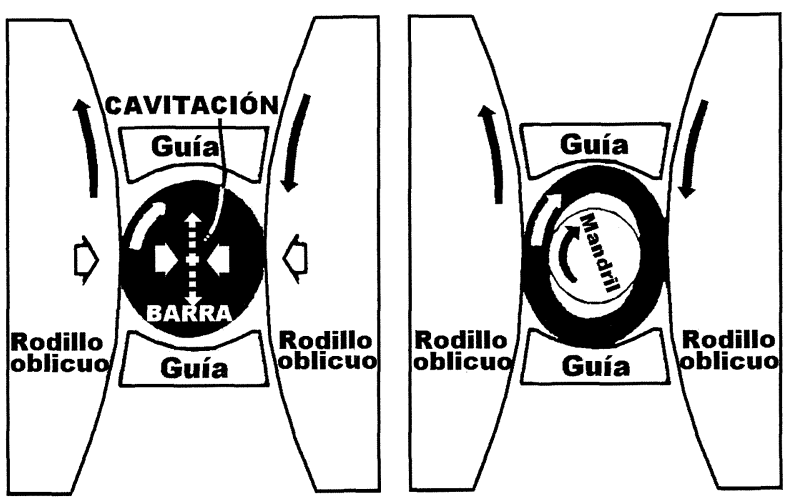

Figura 2. Estado de tensiones en el centro del tocho.

Figure 2. Stress state in the centre of the round billet.

asistida por el proceso de laminación transversal que ocurre en la zona de alimentación (Fig. 3).

Sin embargo, aun bajo estas consideraciones pueden generarse defectos asociados a la aparición prematura de la cavidad central. Esto, en general, es causa de rechazo de productos.

Es relevante considerar que tanto la cavitación en el proceso de laminación transversal como la aparición de la fractura central representan fenómenos no muy bien comprendidos hasta el momento. No obstante, se asume que tiene lugar en la zona de alimentación que conforman los cilindros, como consecuencia de la intensa deformación generada en dicha región durante la laminación transversal del tocho.

Existen, básicamente, dos tipos de fracturas centrales:

- Las que tienen lugar antes de que una sección transversal dada del tocho alcance la punta de perforación y que producirán defectos.

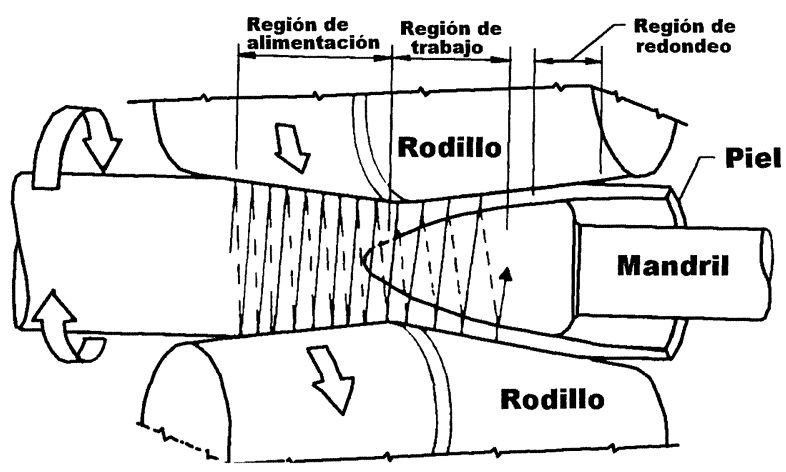

Figura 3. Avance helicoidal del tocho a través de los cilindros.

Figure 3. Screw movement of the billet through the rolling rolls.
- Aquellas que tienen lugar en la nariz de la punta de perforación y que, como consecuencia de su presencia, pueden resultar en tubos sanos.

La aparición de uno u otro tipo de rotura axial no es de simple predicción, no obstante lo cual es fácil de imaginar que todos aquellos factores tanto mecánicos como metalúrgicos que alteren las condiciones de perforado tendrán fuerte influencia sobre los mismos.

En este trabajo se propone estudiar las características de la deformación en caliente de algunos aceros producidos mediante la combinación de procesos de colada continua - laminador perforador. En particular, en lo referido a la ductilidad en caliente y su relación con la susceptibilidad a la rotura axial. Se parte del empleo del ensayo de torsión en caliente, frecuentemente propuesto como una herramienta experimental válida para evaluar este tipo de propiedades en relación con los procesos de fabricación de tubos sin costura ${ }^{[1-6]}$.

En particular, se presentan los resultados obtenidos al ensayar por torsión en caliente probetas extraídas de distintas zonas de una barra de colada continua, a fin de evaluar su comportamiento en el ámbito de temperaturas y velocidades de deformación típicas de las operaciones de perforado.

\section{MATERIALES Y PROCEDIMIENTO EXPERIMEN- TAL}

Con el fin de caracterizar la ductilidad en caliente de las distintas zonas que componen la sección transversal de una barra de colada continua, se han llevado a cabo ensayos de torsión en caliente sobre probetas extraídas de distintas regiones de la misma, según lo esquematizado en la figura 4 . Cabe mencionar que se consideran temperaturas en el rango entre $900^{\circ} \mathrm{C}$ y $1200^{\circ} \mathrm{C}$ y una velocidad de deformación de $4 \mathrm{~s}^{-1}$.

En la tabla I, se presenta la composición química del material en estudio.

Cabe mencionar que, previamente al ensayo, las probetas fueron sometidas a un tratamiento de precalentamiento a $1.250^{\circ} \mathrm{C}$ durante $5 \mathrm{~min}$. con el fin de lograr la homogeneización de la estructura.

Tabla I. Composición química del acero

Table I. Chemical composition of the steel 


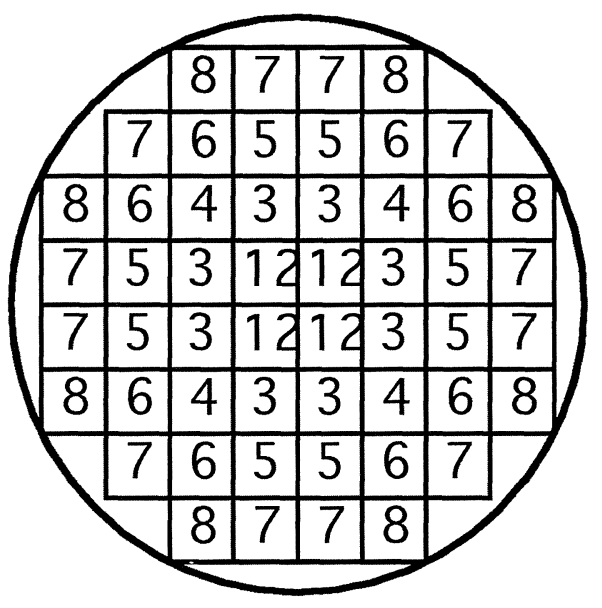

Figura 4. Esquema de las zonas de extracción de las probetas en el tocho.

Figure 4. Scheme of the locations for sample extraction in the round billet.

Las curvas de ductilidad han sido determinadas para cada una de las zonas previamente definidas.

\section{RESULTADOS}

En la figura 5 se presentan los resultados sobre de la caracterización de cada una de las zonas de la barra considerada. Cabe aclarar que cada una de estas zonas corresponde a la sección transversal de la misma. Es relevante mencionar que los datos de ductilidad consignados corresponden a valores corregidos por aumento de la temperatura, a causa de la deformación en el material.

Durante el ensayo se produce un paulatino aumento de la temperatura como consecuencia de la energía entregada por deformación y que, en el ca-

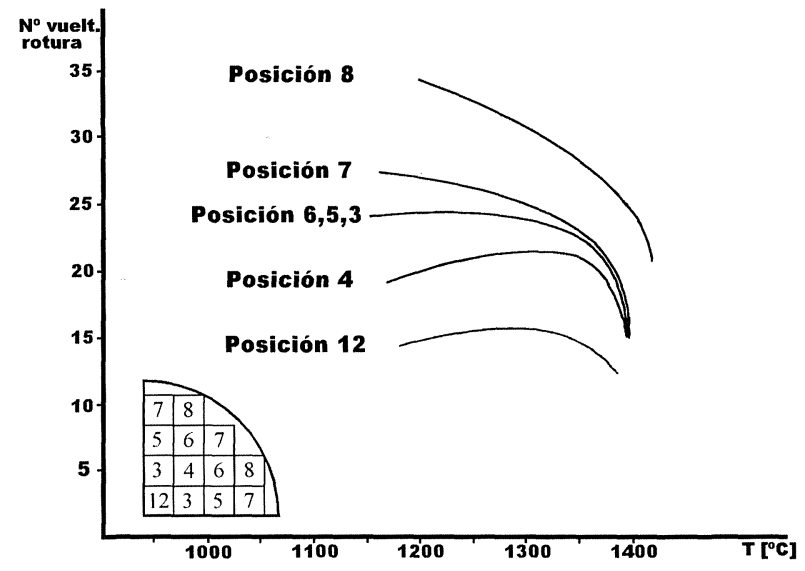

Figura 5. Curvas de ductilidad en caliente con velocidad de deformación de $4 \mathrm{~s}^{-1}$.

Figure 5. Hot ductility curves at $4 \mathrm{~s}^{-1}$ strain rate. Rev. Metal. Madrid Vol. Extr. (2005) 127-130 so de altas velocidades de ensayos utilizadas, no puede ser totalmente disipada. En la figura 6 se puede observar el aumento de temperatura a causa de la deformación en el material. Los datos corregidos surgen de sumar a la temperatura de inicio del ensayo, el aumento registrado. La figura 7 permite notar el desplazamiento que sufren las curvas de ductilidad después de efectuada la corrección.

No obstante, tal como se ha observado en la figura 5, se pueden apreciar las diferencias de comportamiento asociadas a las distintas zonas de la barra.

Se desprende de los resultados obtenidos, que la máxima ductilidad parece asociada con la región más próxima a la zona acoquillada, en tanto que la zona 12 presenta menor ductilidad. Entre estas dos

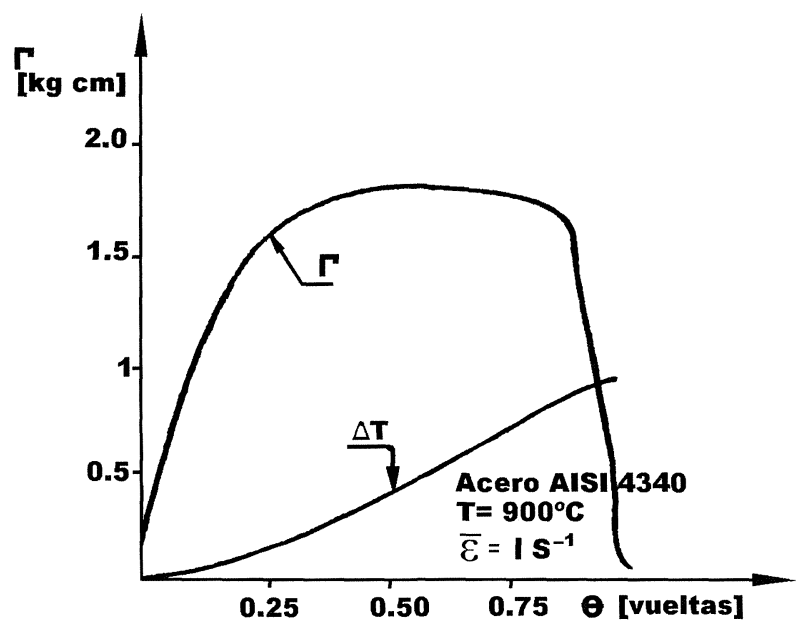

Figura 6. Curva de ensayo de torsión a rotura con velocidad de deformación $1 \mathrm{~s}^{-1}$ y a $900^{\circ} \mathrm{C}$.

Figure 6. Torsion test curve using a $1 \mathrm{~s}^{-1}$ strain rate at 900 ${ }^{\circ} \mathrm{C}$.

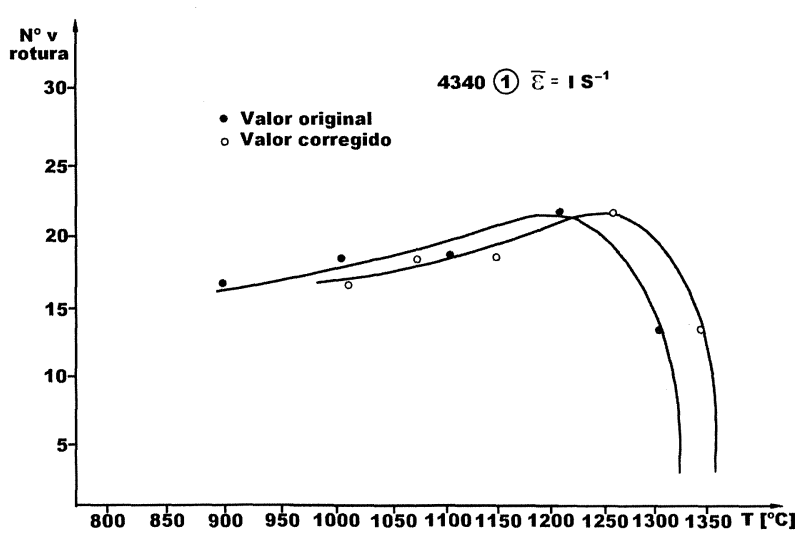

Figura 7. Curvas de ductilidad obtenidas a temperatura de base y por corrección con aumento de temperatura.

Figure 7. Ductility curves obtained at base temperature considering temperature correction. 
regiones límite, respecto a la ductilidad, se ubican las restantes zonas con ductilidad decreciente desde la periferia hacia el centro de la barra ${ }^{[7]}$.

En la zona 4, no sucede el mismo comportamiento, hecho posiblemente asociado a que la estructura es de transición de granos columnares a equiaxiados ${ }^{[8]}$.

La temperatura de máxima ductilidad, tiende a ubicarse en el entorno de $1.250^{\circ} \mathrm{C}$ a $1.315^{\circ} \mathrm{C}$, si se consideran las zonas 12 y 6 , excepto en las zonas 7 y 8.

Parece interesante resaltar aquí la importancia que puede asumir la forma de la curva de ductilidad por cuanto, si bien es cierto que la zona 4 , por ejemplo, presenta una temperatura de ductilidad máxima superior a la de las zonas 3,5 y 7 a 1.315 ${ }^{\circ} \mathrm{C}$ vs $1.250{ }^{\circ} \mathrm{C}$, esta última región muestra un comportamiento mucho menos dependiente de la temperatura que la zona 4, para temperaturas descendentes.

Con relación a este tema, podría definirse, además, una zona de sensible disminución de la ductilidad, en el rango de temperaturas entre $1.350^{\circ} \mathrm{C}$ y $1.375^{\circ} \mathrm{C}$, para lo cual es fácil apreciar el marcado descenso del número de vueltas a la rotura que experimentan las muestras, incluida la acoquillada. Así, también podría definirse una temperatura de ductilidad nula para un número de vueltas a la rotura igual a cero, que en este caso sería de $1.400{ }^{\circ} \mathrm{C}$.

De acuerdo a la literatura, la correcta elección de la temperatura óptima de perforado ejerce una importante influencia, frecuentemente coincidente con la máxima ductilidad obtenida por torsión en caliente ${ }^{\left[4 y^{5}\right]}$. Experiencias llevadas a cabo sobre barras de colada continua pertenecientes a un mismo grado de acero y laminadas transversalmente a tres niveles de temperaturas diferentes, entre $30 \mathrm{y}$ $35{ }^{\circ} \mathrm{C}$ por encima de la temperatura normal de procesado, 30 y $35^{\circ} \mathrm{C}$ por debajo y, finalmente, a la citada temperatura, muestran, en el primer grupo, un creciente número de tubos con defectos internos $^{[9}$ y 10$]$

En estos casos, han sido asociados determinados defectos con el hecho de que antes que el tocho alcance la punta de perforación, su temperatura promedio no es sólo función del ciclo de calentamiento a que ha sido sometido en el horno, sino que ésta se verá fuertemente alterada por el recalentamiento causado por el trabajo redundante más la fricción que tiene lugar en la zona de alimentación. De esta manera la temperatura podría superar incluso los $1.370^{\circ} \mathrm{C}$, de manera tal que la aparición de defectos podría ser directamente atribuida a la pérdida de ductilidad ${ }^{[10]}$.

De este modo, queda expuesta la idea central del trabajo, que manifiesta la importancia que reviste no sólo conocer la curva de ductilidad máxima sino, además, la forma que adopta la misma.

\section{CONCLUSIONES}

A partir de los resultados obtenidos se puede decir que:

- En base a la curva de ductilidad en caliente, obtenida sobre las distintas zonas de la barra de colada continua, se pudo comprobar que el empobrecimiento de la ductilidad ocurre desde la periferia hacia el centro de la barra.

- Se pudo determinar, en cada caso, la temperatura de máxima ductilidad de cada zona de la barra, las mismas se hallan comprendidas entre $1.250^{\circ} \mathrm{C}$ y $1.315^{\circ} \mathrm{C}$.

- La zona de pérdida brusca de ductilidad está comprendida en el rango de temperaturas entre $1.350^{\circ} \mathrm{C}$ y $1.375^{\circ} \mathrm{C}$.

\section{REFERENCIAS}

[1] D.E.R. Hughes, Rev. JISI, (1952) 214-222.

[2] J. Dauverge, M. Pelabon y J. Ivanel, Rev. Metal. Madrid 4 (1954) 254-264.

[3] F. Morozumi, Rev. Trans ISIJ 8 (1968) 14-24.

[4] G.S. Diegel y C.J. RosA, Rev. Metall. Bd. 73, H5, (1982) 335-344.

[5] J.J. Burroni, H. Posdena, G.R. Carfi, J. Tormo, J. Ruzzante y A. Hey, $11^{\circ}$ Seminario de Laminación IAS, Mar del Plata, (1985).

[6] W. Kubinski, Z. KuZMinski y J. StarowicZ, Rev. Bauder, Bleche Rohre, 27 (1986) 59-62.

[7] J. Piestch y P. Thieven, Rev. MPT 2 (2003) 52-60.

[8] J. Bellto y M. Hugo, Rev. Metal. Madrid (1966) 7-16.

[9] E. Erman, J. App. Met. 4 (1987) 331-341.

[10] A.N. KiRICHENCO, Rev. Stal. (1961) 594-604. 